\title{
Epileptic, organic and genetic vulnerabilities for timing of the development of interictal psychosis
}

Naoto Adachi, Nozomi Akanuma, Masumi Ito, Masaaki Kato, Tsunekatsu Hara, Yasunori Oana, Masato Matsuura, Yoshiro Okubo and Teiichi Onuma

\section{Background}

Age at the first psychotic episode and an interval between the onset of epilepsy and that of psychosis reflect developmental processes of interictal psychosis. However, factors relating to these indices remain unknown.

\section{Aims \\ To identify clinical variables that are associated with the timing of the development of interictal psychosis}

\section{Method}

In 285 adults with epilepsy with interictal psychosis, effects of epileptic (epilepsy type), organic (intellectual functioning) and genetic (family history of psychosis) variables on timing of the development of psychosis were examined.

\section{Results}

The mean interval between the onset of epilepsy and that of psychosis was 14.4 years. Some psychosis occurred within a few years of the first seizure. Generalised epilepsy, normal intellectual function and a positive family history of psychosis were associated with early onset of psychosis.

\section{Conclusions}

Early development of interictal psychosis in people with epilepsy may reflect other individual vulnerabilities to psychosis rather than epilepsy-related damage.

\section{Declaration of interest}

None.
Interictal psychosis in epilepsy was first studied systematically by Slater and his colleagues. ${ }^{1}$ They reported three main pieces of evidence to delineate interictal psychosis (called schizophrenialike psychosis in the paper) as a distinct entity from schizophrenia: psychopathological characteristics, psychosis occurring after the development of epilepsy and no genetic loading for schizophrenia. Subsequent studies have formed a general consensus that interictal psychosis is mainly related to various epilepsy-related factors such as type of epilepsy, seizure types and laterality and locality of electroencephalogram (EEG) abnormalities, rather than nonspecific demographic factors. ${ }^{2}$ However, studies on interictal psychosis have shown contradictive findings that some of the demographic characteristics such as intellectual function ${ }^{3,4}$ and family history of psychosis ${ }^{5}$ were associated with occurrence of interictal psychosis. This is similar to the positive associations between these demographic factors and a high risk of functional psychoses such as schizophrenia. Using a comprehensive, multicentre database of patients suffering from epilepsy with and without psychosis, our group has found that interictal psychosis occurred more frequently in individuals with certain risk factors, including partial epilepsies, complex partial seizures, generalised tonic-clonic seizures, earlier onset of epilepsy and borderline intellectual function. ${ }^{6}$ Most of these risk factors were also common in different types of epilepsy psychoses (e.g. interictal, postictal and bimodal psychoses), ${ }^{7}$ but some factors historically known as risk factors for interictal psychosis were not extracted with multivariate analyses because they overlapped or interacted with others. ${ }^{6-8}$

Age at the time of the first psychotic episode and the time interval between the onset of epilepsy and that of psychosis are key elements of studies in interictal psychosis, as these age-related variables likely reflect neurodevelopmental and/or neurodegenerative processes in the brain. ${ }^{9}$ Indeed, Slater et al ${ }^{1}$ showed that patients with interictal psychosis tend to suffer their first seizure in early adolescence, with psychosis developing in their late twenties or thirties (approximately 15 years after the onset of epilepsy). They interpret the long interval, during which epilepsy and its consequences could cause further damage to the brain, as a preparatory period for generation of psychosis. Whereas many studies have reported similar age-related variables, ${ }^{2}$ some have suggested the interval is an artefact as a result of the wide range of distribution of time intervals and to the tendency of a shorter interval in individuals with late-onset epilepsy. ${ }^{10,11}$ In our previous study, ${ }^{12}$ age at onset of psychosis in a subgroup of patients with chronic interictal psychosis was comparable with that in those with schizophrenia, whereas the age at onset was more advanced in the whole group of patients with interictal psychosis (both episodic and chronic). We also showed no difference between various types of partial epilepsies in age at onset of psychosis and in time intervals. ${ }^{8}$ However, few studies have examined the contributions of the other clinical factors to age-related variables; thus, it remains unknown whether particular clinical factors are related to the timing of development of interictal psychosis. In the current study, we investigated the timing of development of interictal psychosis in association with epilepsy-related and demographic characteristics in a large cohort of patients with interictal psychosis.

\section{Method}

\section{Definition of interictal psychosis}

In our study, psychosis was defined as the presence of hallucinations, delusions or a limited number of severe abnormalities of behaviour in accordance with the ICD-10. ${ }^{13}$ The operational criteria for interictal psychosis were as follows: the psychosis developed after the onset of epilepsy; $;^{1,14,15}$ the psychotic episodes occurred with no distinct antecedent seizures when the patient was seizure-free or between habitual seizures; ${ }^{6,7}$ psychotic episodes lasted $24 \mathrm{~h}$ or more in a state of full consciousness. Interictal 
psychosis included chronic schizophrenia-like psychosis (at least one episode lasting 1 month or more) and brief (acute, episodic) interictal psychosis (all episodes resolved within 1 month). ${ }^{1,16-18}$ Postictal psychosis, which occurred within 7 days after a decisive seizure or cluster of seizures, ${ }^{7,17,19}$ and ictal psychotic phenomenon ${ }^{17}$ were excluded.

\section{Participants}

All participants met the criteria for epilepsy as set forth in the 1989 International Classification of Epilepsies and Epileptic Syndromes. ${ }^{20}$ The participants all attended one of five institutions with adult epilepsy clinics: National Centre Hospital for Mental, Nervous, and Muscular Disorders; Nihon University Hospital; Tokyo Medical University Hospital; Tokyo Medical and Dental University Hospital; or Komagino Hospital. The five epilepsy clinics cover the greater Tokyo area of a population of approximately 35 million as the main neuropsychiatric institutions for adults with epilepsy. In addition, the National Centre Hospital is the only institution in the country that has a neuropsychiatric in-patient unit dedicated to patients with epilepsy, accepting tertiary referrals from outside the catchment area. Since August 1996, these epilepsy clinics have maintained a collaborative database designed specifically for epilepsy psychosis. ${ }^{6-8,12}$ Our previous studies ${ }^{6-8,12}$ were based on the database with patients who had been registered until the end of 1996. The current study was conducted with a data-set entered until December 2000, with a total of 313 patients with epilepsy and interictal psychotic episodes being identified. To focus on interictal psychosis, 19 patients with bimodal psychosis, who exhibited both interictal and postictal psychoses in distinct periods, ${ }^{7}$ were excluded from the study. Five patients with epilepsy resulting from a neurodegenerative disorder and four without sufficient clinical information regarding the epilepsy were also excluded. Consequently, 285 patients with interictal psychosis were enrolled in the study. No participants showed evidence of substance misuse, dementing process or a recent progressive space-occupying lesion.

\section{Variables studied}

We investigated the following variables:

(a) age at the time of investigation;

(b) gender;

(c) family history of psychosis, i.e. any psychotic disorder (schizophrenia, other paranoid disorder, acute transient psychosis, etc.) in a first-degree relative, according to the Japanese version of the Family History Research Diagnostic Criteria; ${ }^{21}$

(d) age at the onset of epilepsy, i.e. age at the time of the first afebrile seizure;

(e) type of epilepsy based on ictal symptoms, EEG findings and neuroimaging in accordance with the International Classification of Epilepsies and Epileptic Syndromes ${ }^{20}$ (i.e. localisation-related epilepsies and generalised epilepsies, including idiopathic and symptomatic);

(f) intellectual functioning: impaired (full-scale IQ on the Wechsler Adult Intelligence Scale-Revised ${ }^{22}$ of 70 or below), borderline (of 71-84), or normal (of 85 or above) in accordance with the DSM-IV; ${ }^{23}$

(g) age at onset of psychosis (i.e. age at the time of the first psychotic episode); (h) time interval between the onset of epilepsy and that of psychosis, calculated as age at onset of psychosis minus age at onset of epilepsy.

As different neuroimaging techniques were used during different time periods and by each institution, neuroimages were used only for diagnostic information. Diagnoses and evaluations were made by consultant neuropsychiatrists qualified in both psychiatry and epileptology. The study was approved by the ethics committees of the institutions.

\section{Data analysis}

Differences in linear variables (ages) for the categorical variables (gender, epilepsy type and family history) were subjected to analysis of variance (ANOVA). Correlation between categorical variables was examined by means of the chi-squared test or Fisher's exact test. Correlations between linear or rank-order variables (intellectual functioning) were examined by means of simple regression analysis or Spearman's rank-order correlation coefficient. Because age at the time of examination was correlated significantly with the other age-related variables (age at the onset of epilepsy $(r=0.39, \quad P<0.0005)$, time interval $(r=0.31$, $P<0.0005)$, and age at the onset of psychosis $(r=0.62$, $P<0.0005)$ ), the weighted least squares procedure (weighted by age at the time of examination) was applied. ${ }^{12}$ A $P$-value of $<0.05$ was considered significant. All statistical analyses were performed with the Statistical Package for Social Sciences (SPSS) 14.0 for Windows.

\section{Results}

Clinical characteristics of the 285 patients with interictal psychosis were as follows: mean age at the time of examination was 40.7 years (s.d. $=12.8$, range $19-76$, median 39). There were 146 men and 139 women. A total of 236 patients had localisation-related epilepsies and 49 generalised epilepsies (34 with idiopathic and 15 with symptomatic generalised epilepsies). With respect to estimated aetiologies of epilepsy, there were 22 patients with central nervous system infections, 26 with birth complications (including cerebral palsy), 15 with head trauma, 7 with brain tumours, 16 with migration disorders or other malformation, 5 with vascular disorders, and pathogenesis was unknown for the remaining 194 patients. Intellectual function was normal in 140 patients, borderline in 55, and impaired in 90. There were 244 patients with chronic schizophrenia-like psychosis, 27 with brief interictal psychosis and 14 with interictal psychosis of unknown duration. Twenty-one patients had a family history of psychosis.

Distributional relations between the patients' characteristics studied were as follows: gender and intellectual functioning $\left(\chi^{2}=2.6, P=0.280\right)$, gender and epilepsy type (129 men and 107 women with localisation-related epilepsies, 17 men and 32 women with generalised epilepsies; $\chi^{2}=5.7, P=0.017$ ), gender and family history of psychosis $\left(\chi^{2}=0.11, P=0.736\right)$, intellectual functioning and epilepsy type $\left(\chi^{2}=4.1, P=0.126\right)$, intellectual functioning and family history of psychosis $\left(\chi^{2}=0.44, P=0.802\right)$, and epilepsy type and family history of psychosis $\left(\chi^{2}=0.06, P=0.767\right)$.

Age-related factors observed were as follows: mean age at onset of epilepsy was 11.7 years ( $\mathrm{s} . \mathrm{d}=8.0$, range $0-51$, median 11 ), mean age at onset of psychosis was 26.1 years (s.d. $=9.6$, range $12-65$, median 24) and the mean time interval between the onset of epilepsy and that of psychosis was 14.4 years (s.d. $=9.3$, range 0-51, median 13). Distribution of the time intervals for the entire patient group are shown in Fig. 1. Age at onset of psychosis correlated significantly with that of epilepsy $(r=0.47$, $P<0.0005)$ and with the time interval $(r=0.64, P<0.0005)$. 


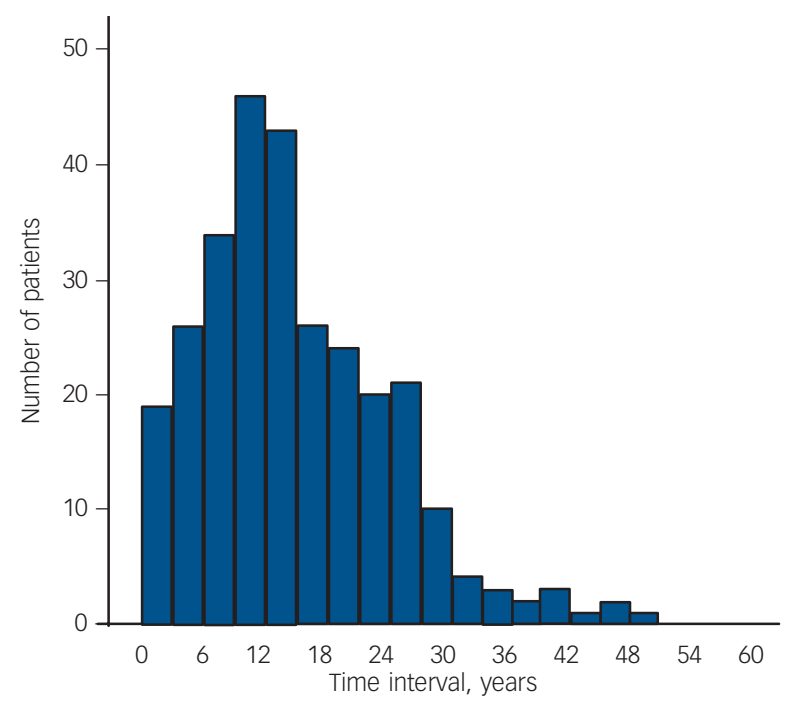

Fig. 1 Distribution of the time intervals (years) between the onset of epilepsy and that of interictal psychosis (mean 14.4 years, s.d. $=9.2$, range $0-51$, median 13 ).

The time interval was 3 years or less in 31 patients $(10.9 \%), 5$ years or less in 45 $(15.8 \%), 10$ years or less in $101(38.6 \%)$

The time interval also correlated significantly with age at onset of epilepsy $(r=-0.38, P<0.0005)$.

The estimated marginal means of age at onset of epilepsy, age at onset of psychosis and the time interval for each variable are shown in Table 1. The time interval and age at onset of psychosis differed significantly between epilepsy types: interictal psychosis developed at an earlier age and with a shorter interval in patients with generalised epilepsies, in particular with idiopathic generalised epilepsies, than those in patients with localisation-related epilepsies. Intellectual functioning correlated significantly with age at onset of epilepsy and the time interval: the onset of epilepsy was earlier and the interval was longer in those patients with intellectual disturbances than in those without. The onset of psychosis was significantly earlier in patients with a family history of psychosis than in those without.

We carried out further analyses on the participants with localisation-related epilepsies $(n=236)$ and obtained similar tendencies: intellectual functioning correlated significantly with age at onset of epilepsy $(r=0.293, P<0.0005$; impaired, estimated marginal mean 9.3 (s.e. $=1.1$ ), borderline $11.3($ s.e. $=1.2$ ), normal 15.3 (s.e. $=0.8)$ ), with age at onset of psychosis $(r=0.128$, $P=0.049$; impaired $26.3($ s.e. $=1.4)$, borderline 29.8 (s.e. $=1.6)$, normal $30.0($ s.e. $=1.0))$ or with time interval $(r=-0.157$, $P=0.016$; impaired 17.0 (s.e. $=1.2$ ), borderline $18.5($ s.e. $=1.4$ ), normal $14.6($ s.e. $=0.9)$ ). Likewise, in the family history of psychosis of the participants with localisation-related epilepsies, the estimated marginal mean age at onset of psychosis also differed significantly $(F=5.45, \quad P=0.020 ; \quad$ positive 22.7 $($ s.e. $=2.8)$, negative $29.4($ s.e. $=0.8)$ ). However, there was no significant difference in age at onset of epilepsy $(F=1.33$, $P=0.250$; positive $10.3($ s.e. $=2.3)$, negative $13.0($ s.e. $=0.6)$ ) or in time interval $(F=2.33, P=0.129$; positive 12.4 (s.e. $=2.5)$, negative $16.4($ s.e. $=0.7)$ ).

\section{Discussion}

In the current study, age at onset of interictal psychosis and time interval between onset of epilepsy and that of psychosis varied

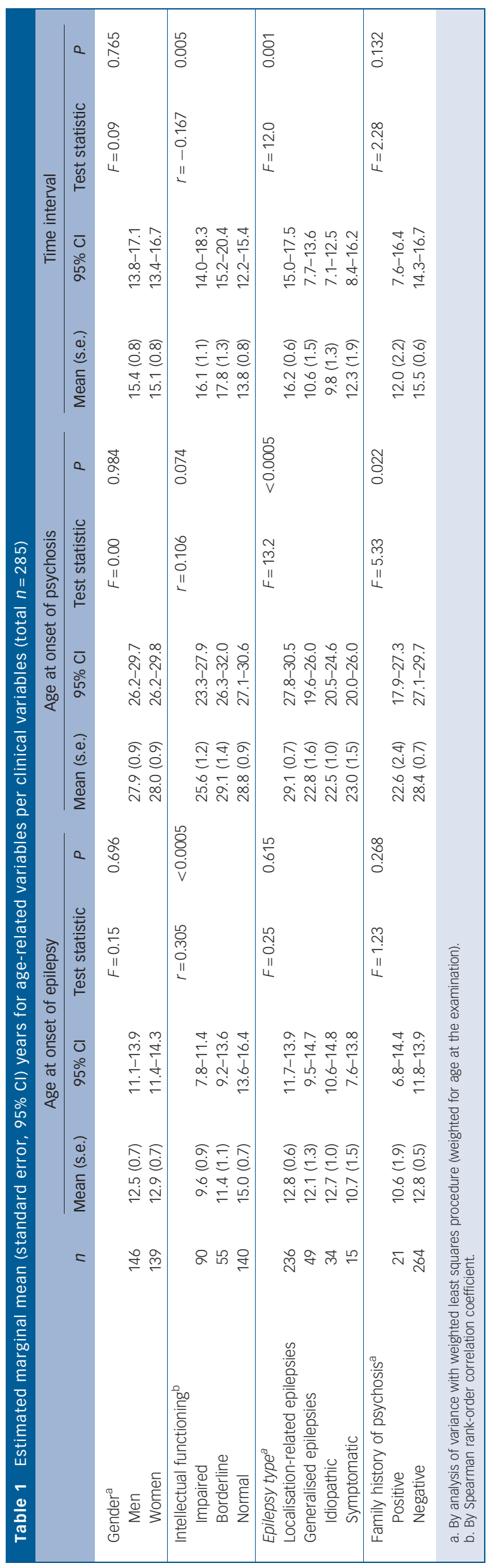


considerably. Participants with generalised epilepsy, normal intellectual function or a positive family history of psychosis tended to show an early onset of interictal psychosis.

\section{Distribution of the time interval}

The mean interval between the onset of epilepsy and that of psychosis was 14.4 years, consistent with previously reported data. ${ }^{1,2}$ This interval varied widely among patients, not showing a simple bell-curve distribution. The wide variation may be in part accounted for by the cumulative effects of various epilepsy-related factors on the development of interictal psychosis, i.e. repeated seizures, frequent epileptic discharges in the brain, adverse effects of anti-epileptic drugs and psychosocial stress. ${ }^{2,18}$ However, it is important to note that interictal psychosis developed in a considerable number of patients shortly after their first epileptic event (within a few years). Indeed, this fact has been described in previous studies. ${ }^{1,11}$ It is not likely that such quick development of interictal psychosis is as a result of the epilepsy-related process alone. There is little evidence that occurrence of interictal psychotic symptoms is precipitated by a higher impact of particular epilepsy processes (e.g. excessive seizures and extensive epileptogenesis), ${ }^{17}$ although severe epilepsy can be a risk factor for the development of psychosis. Thus, in addition to the epilepsy-related process, the presence of certain preparatory conditions, such as individual vulnerabilities to psychosis $^{24}$ that may be common to organic psychoses or even functional psychoses, may play a role in generating psychotic symptoms in individuals with epilepsy.

\section{Epilepsy type}

The interval between onset of epilepsy and that of psychosis was significantly shorter in patients with generalised epilepsies than in those with localisation-related epilepsies, with the onset of epilepsy being comparable among these two groups. Patients with generalised epilepsies, unlike those with localisation-related epilepsies, tend to have fewer epilepsy (organic)-related risk factors for psychosis, i.e. no distinct brain insult, low seizure frequency, simple medications and normal cognitive functioning, which may be associated with a reduced frequency of development of interictal psychosis. ${ }^{2,6}$ It is possible that patients with generalised epilepsies in whom interictal psychosis develops might be affected by non-epileptic precipitators of psychosis. This may be similar to the difference between patients with schizophrenia and those with epilepsy; psychosis is observed at a more advanced age in patients with epilepsy than in patients with schizophrenia that does not involve distinct brain damage. ${ }^{12}$ Among patients with generalised epilepsies, only those with a strong vulnerability may suffer interictal psychosis at an early age regardless of acquired brain insults because of epilepsy.

\section{Intellectual functioning}

Our patients with normal intellectual functioning exhibited interictal psychosis sooner after the onset of epilepsy. This finding was also seen in the subgroup of participants with localisationrelated epilepsies only. Impaired intellectual function is often associated with severe epilepsy and brain damage, ${ }^{25}$ although it is also observed in people without such conditions. ${ }^{26}$ Functional psychosis develops two to three times more frequently in people with impaired intellectual functioning than is reported in the general population. ${ }^{26,27}$ Moreover, psychosis develops $1.3-4.7$ times more frequently in patients with epilepsy with impaired intellectual functioning than in those without. ${ }^{7}$ In contrast, normal intellectual functioning usually suggests having less brain damage and is not related to increased risks for the development of psychosis. Why do patients with a lower risk suffer psychosis earlier than those at a higher risk? Again, psychosis may develop more quickly in patients with normal intellectual functioning who have strong congenital vulnerabilities to psychosis than in those with acquired organic precipitators, i.e. intellectual dysfunction and epilepsy, but without such vulnerabilities.

\section{Family history of psychosis}

We have shown that interictal psychosis develops at an earlier age in patients with a family history of psychosis than in those without. A genetic tendency towards psychosis in patients with epilepsy has long been underestimated ${ }^{2}$ since Slater's initial study. ${ }^{1}$ However, large studies have shown that genetic factors play a significant role in the development of psychosis in patients with epilepsy. ${ }^{5,6}$ These findings appear to be similar to those found in functional psychosis (i.e. schizophrenia); people with a positive family history tend to have a higher risk of psychosis and to exhibit their first psychotic symptoms earlier than those without. ${ }^{28,29}$ A positive family history of psychosis may be a universal risk factor for developing psychosis, and it appears to reflect, at least in part, a congenital vulnerability to psychosis. ${ }^{23}$ Even in patients with epilepsy and a positive family history of psychosis, psychotic symptoms are likely generated sooner regardless of acquired risk factors related to either epilepsy or brain damage.

\section{Study limitations}

Some limitations should be considered in relation to the current study. Analysis of age at onset of psychosis in patients with epilepsy is subject to some methodological issues. ${ }^{10}$ Because epilepsy psychosis was defined operationally as psychosis developing after the onset of epilepsy in accordance with Slater \& Roth's definition, ${ }^{14}$ two patient groups were excluded: patients in whom psychosis developed before epilepsy ${ }^{17}$ and patients in whom novel psychoses will develop after the time of the investigation or who died before the possible development of psychosis. However, neither group would have been large enough to markedly influence mean age at onset of psychosis or the mean time interval between onsets of the two disorders. Neither of these omissions explains the significant differences in age at onset of psychosis or in the onset interval between patients with particular clinical characteristics. In addition, despite the large cohort of participants with interictal psychosis, the number of patients in whom particular factors were analysed, such as a positive family history of psychosis and generalised epilepsies, was insufficient to produce strong statistical power. Factors that we did not consider may be associated with age-related factors, but would not have affected the result of our study. Although our findings point to the effects of certain vulnerabilities to psychosis (reflected by a positive family history), it is still unclear what these vulnerabilities are. Evidence supporting such vulnerability concepts is scarce, even for patients with functional psychosis. ${ }^{24}$

Results of the current study show some relationship between age at onset of interictal psychosis and several clinical variables that may reflect individual vulnerabilities. These vulnerabilities, in addition to epilepsy-related deficits, can affect the generation of interictal psychosis independently or interactively. Further comprehensive studies to confirm such vulnerabilities are required. 
Naoto Adachi, MD, Adachi Mental Clinic, Sapporo, and Depatment of Psychiatry, National Centre Hospital for Mental, Nervous and Muscular Disorders, Kodaira; Nozomi Akanuma MD, Department of Psychiatry, National Centre Hospital for Mental, Nervous and Muscular Disorders, Kodaira, and Department of Neuropsychiatry, School of Medicine, Tokyo Medical and Dental University, Tokyo; Masumi Ito, MD, Masaaki Kato, MD, Department of Psychiatry, National Centre Hospital for Mental, Nervous and Muscular Disorders, Kodaira; Tsunekatsu Hara, MD, Komagino Hospital, Hachioji; Yasunori Oana, MD, Department of Neuropsychiatry, School of Medicine, Tokyo Medical University, Tokyo; Masato Matsuura, MD, Department of Neuropsychiatry, Nihon University School of Medicine Tokyo; Yoshiro Okubo, MD, Department of Neuropsychiatry, School of Medicine, Tokyo Medical and Dental University, Tokyo; Teiichi Onuma, MD, Department of Psychiatry, National Centre Hospital for Mental, Nervous and Muscular Disorders, Kodaira, Japan

Correspondence: Naoto Adachi, Adachi Mental Clinic, Kitano 7-5-12, Kiyota, Sapporo 004-0867, Japan. Email: adacchan@tky2.3web.ne.jp

First received 30 Jun 2009, final revision 5 Aug 2009, accepted 11 Nov 2009

\section{References}

1 Slater E, Beard AW. The schizophrenia-like psychoses of epilepsy. i. Psychiatric aspects. Br J Psychiatry 1963; 109: 95-112.

2 Trimble MR, Schmitz B. The psychoses of epilepsy/schizophrenia. In Epilepsy: A Comprehensive Textbook (eds J Engel Jr, TA Pedley): 2071-81. LippincottLeven, 1997.

3 Mellers J, Toone BK, Lishman WA. A neuropsychological comparison of schizophrenia and schizophrenia-like psychosis of epilepsy. Psychol Med 2000; 30: 325-35.

4 Matsuura $\mathrm{M}$, Adachi $\mathrm{N}$, Muramatsu $\mathrm{R}$, Kato $\mathrm{M}$, Onuma $\mathrm{T}$, Okubo $\mathrm{Y}$, et al. Intellectual disability and psychotic disorders of adult epilepsy. Epilepsia 2005; 46 (suppl 1): 11-4

5 Qin P, Xu H, Laursen TM, Vestergaard M, Moriensen PB. Risk for schizophrenia and schizophrenia-like psychosis among patients with epilepsy: population based cohort study. BMJ 2005; 331: 23-5.

6 Adachi N, Matsuura M, Okubo Y, Oana Y, Takei N, Kato M, et al. Predictive variables of interictal psychosis in epilepsy. Neurology 2000; 55: 1310-4.

7 Adachi N, Matsuura M, Hara T, Oana Y, Okubo Y, Kato M, et al. Psychoses and epilepsy: are interictal and postictal psychoses distinct clinical entities? Epilepsia 2002; 43: 1574-82.

8 Adachi N, Onuma T, Hara T, Matsuura M, Okubo Y, Kato M, et al. Frequency and age-related variables in interictal psychoses in localization-related epilepsies. Epilepsy Res 2002; 48: 25-31.

9 Weinberger DR. Implications of normal brain development for pathogenesis of schizophrenia. Arch Gen Psychiatry 1987; 44: 660-9.

10 Stevens JR. Psychiatric implications of psychomotor epilepsy. Arch Gen Psychiatry 1966; 14: 461-71.
11 Parnas J, Korsgaad S, Krautwald O, Jensen PS. Chronic psychosis in epilepsy. A clinical investigation of 29 patients. Acta Psychiatr Scand 1982; 66: 28293.

12 Adachi $\mathrm{N}$, Hara $\mathrm{T}$, Oana $\mathrm{Y}$, Matsuura $\mathrm{M}$, Okubo $\mathrm{Y}$, Akanuma $\mathrm{N}$, et al. Difference in age of onset of psychosis between epilepsy and schizophrenia. Epilepsy Res 2008; 78: 201-6.

13 World Health Organization. The ICD-10 Classification of Mental and Behavioural Disorders: Clinical Descriptions and Diagnostic Guidelines. WHO, 1992.

14 Pond DA. Psychiatric aspects of epilepsy. J Indian Med Prof 1957; 3: 1421-51.

15 Slater E, Roth M. Mayer-Gross, Slater and Roth Clinical Psychiatry (3rd edn). Baillière Tindal, 1969.

16 Bruens J. Psychoses in epilepsy. In Handbook of Clinical Neurology, vol.15 (eds P Vinken, GW Bruyn): 593-610. North-Holland Publishing, 1974.

17 Sachdev P. Schizophrenia-like psychosis and epilepsy: the status of association. Am J Psychiatry 1998; 155: 325-36.

18 Adachi N. Tenkan to seishinbyou: rinshou-kenkyu ni okeru kadai. [Epilepsy and psychosis. Issues on clinical research in epilepsy psychosis]. Seishin Shinkeigaku Zasshi 2006; 108: 260-5.

19 Logsdail SJ, Toone BK. Post-ictal psychoses. A clinical and phenomenological description. Br J Psychiatry 1988; 152: 246-52.

20 Commission on Classification and Terminology of the International League Against Epilepsy. Proposal for revised classification of epilepsies and epileptic syndromes. Epilepsia 1989; 30: 389-99.

21 Kitamura T, Shima S, Sakio E, Kato M. Shourei-toushi-hou ni yoru kazokurekikenkyu-shindan-kijun (FH-RDC) no shinraido-kentei. [Reliability Study on Family History-Research Diagnostic Criteria (FH-RDC) by using case vignettes]. Jpn J Soc Psychiatry 1984; 7: 308-12.

22 Wechsler D. The Wechsler Adult Intelligence Scale-Revised. The Psychological Corporation, 1981.

23 American Psychiatric Association. Diagnostic and Statistical Manual of Mental Disorders (4th edn) (DSM-IV). APA, 1994.

24 Ingram RE, Price JM. The role of vulnerability in understanding psychopathology. In Vulnerability to Psychopathology. Risk Across the Life Span (eds RE Ingram, JM Price): 3-19. Guilford Press, 2001.

25 Kirkham F. Epilepsy and mental retardation. In Epilepsy (2nd edn) (eds A Hopkins, S Shorvon, G Cascino): 503-20. Chapman \& Hall, 1995.

26 David AS, Malmberg A, Brandt L, Allebeck P, Lewis G. IQ and risk for schizophrenia: a population-based cohort study. Psychol Med 1997; 158: 103-5.

27 Reid AH. The Psychiatry of Mental Handicap. Blackwell Scientific Publications, 1982.

28 Albus M, Maier W. Lack of gender differences in age at onset of familial schizophrenia. Schizophr Res 1995; 18: 51-7.

29 Nicholson IR, Neufeld RW. A dynamic vulnerability perspective on stress and schizophrenia. Am J Orthopsychiatry 1992; 62: 117-30. 